\title{
THREE-DIMENSIONAL EVOLUTION OF THE PARKER INSTABILITY UNDER A UNIFORM GRAVITY
}

\author{
Jongsoo Kim, ${ }^{1,2}$ S. S. Hong, ${ }^{2}$ Dongsu Ryu, ${ }^{3}$ and T. W. Jones ${ }^{4}$ \\ Received 1998 June 9; accepted 1998 August 18; published 1998 September 11
}

\begin{abstract}
Using an isothermal MHD code, we have performed three-dimensional, high-resolution simulations of the Parker instability. The initial equilibrium system is composed of exponentially decreasing isothermal gas and a magnetic field (along the azimuthal direction) under a uniform gravity. The evolution of the instability can be divided into three phases: linear, nonlinear, and relaxed. During the linear phase, the perturbations grow exponentially with a preferred scale along the azimuthal direction but with the smallest possible scale along the radial direction, as predicted from linear analyses. During the nonlinear phase, the growth of the instability is saturated and flow motion becomes chaotic. Magnetic reconnection occurs, which allows the gas to cross field lines. This, in turn, results in the redistribution of the gas and the magnetic field. The system approaches a new equilibrium in the relaxed phase, which is different from the one seen in two-dimensional works. The structures formed during the evolution are sheetlike or filamentary, whose shortest dimension is radial. Their maximum density enhancement factor relative to the initial value is less than 2. Since the radial dimension is too small and the density enhancement is too low, it is difficult to regard the Parker instability alone as a viable mechanism for the formation of giant molecular clouds.
\end{abstract}

Subject headings: instabilities — ISM: clouds — ISM: magnetic fields — ISM: structure — MHD

\section{INTRODUCTION}

Under a uniform gravity, a vertically stratified system of interstellar medium (ISM) composed of gas, a magnetic field, and cosmic rays is unstable against perturbations (Parker 1966, 1967). This nature has been called the Parker instability in the astronomical literature. The instability is broken down into two distinct modes: undular and interchange (Hughes \& Cattaneo 1987). The former, whose wavevectors lie in the plane defined by the direction of gravitational field (the vertical direction) and the direction of the unperturbed magnetic field (the azimuthal direction), undulates the azimuthal field lines and induces the gas to slide down along the field lines into "magnetic valleys." The critical adiabatic index for the undular mode without taking into account cosmic rays is $\gamma_{\mathrm{cr}, u}=(1+$ $\alpha)^{2} /(1+1.5 \alpha)$, where $\alpha$ is the ratio of magnetic to gas pressure. The undular mode has a preferred wavelength along the azimuthal direction. The latter interchange mode, whose wavevectors lie in the plane perpendicular to the direction of the unperturbed magnetic field, leaves the direction of the magnetic field unchanged but alters its strength. The critical adiabatic index of the interchange mode is $\gamma_{\mathrm{cr}, i}=1-\alpha$. This mode has a maximum growth rate at infinite wavenumber, indicating it is a Rayleigh-Taylor type. If the wavevectors are allowed to have all three components, then there exists the mixed mode of the undular and interchange modes (Matsumoto et al. 1993). The critical adiabatic index of the mixed mode is $\gamma_{\text {cr, } m}=$ $1+\alpha$.

If three-dimensional perturbations are applied to a system with the effective adiabatic index $\gamma_{\mathrm{cr}, i}<\gamma<\gamma_{\mathrm{cr}, u}$, which is typical of the ISM, the system is unstable to the undular mode but stable to the interchange one. The mixed mode, however,

\footnotetext{
${ }^{1}$ Korea Astronomy Observatory, San 36-1, Hwaam-Dong, Yusong-Ku, Taejon 305-348, Korea; jskim@ @anul.issa.re.kr.

${ }^{2}$ Department of Astronomy, Seoul National University, Seoul 151-742, Korea; sshong@astroism.snu.ac.kr.

${ }^{3}$ Department of Astronomy and Space Science, Chungnam National University, Taejon 305-764, Korea; ryu@ canopus.chungnam.ac.kr.

${ }^{4}$ Department of Astronomy, University of Minnesota, Minneapolis, MN 55455; twj@msi.umn.edu.
}

has a maximum growth rate at vanishing wavelength along the radial direction (Parker 1967), showing the characteristic of the interchange mode. This implies that the interchange mode as well as the undular mode are involved in the three-dimensional development of the Parker instability. It is our understanding that the "magnetic arches," which are formed by the undular mode, are less dense than their surroundings and are now susceptible to the interchange mode.

It has been considered that the Parker instability is one of the plausible mechanisms for the formation of giant molecular clouds (GMCs) (Mouschovias, Shu, \& Woodward 1974; Blitz \& Shu 1980). As the formation mechanism of GMCs, the Parker instability, however, has two problems. One is that, as we have mentioned in the last paragraph, its linear growth rate is a maximum at infinite wavenumber along the radial direction of our Galaxy. This means that chaotic structures rather than ordered large-scale ones are expected to form. The other is that, according to two-dimensional numerical simulations of the Parker instability (Basu, Mouschovias, \& Paleologou 1997), the density enhancement is at most a factor of 2 or so, which is too small.

In this paper, we report the three-dimensional simulations of the Parker instability under a uniform gravity, which are intended to study the mixed mode. Our work is a three-dimensional extension of that of the two-dimensional undular mode by Basu, Mouschovias, \& Paleologou (1996) and Basu et al. (1997). The simulations enable us to answer the following questions on the three-dimensional development of the Parker instability: (i) What are the structures formed in the nonlinear stage? (ii) What is the density enhancement factor? Based on the answers, we address the role of the Parker instability in the formation of GMCs. In addition, the simulations show that there is a final equilibrium state that is different from that obtained in the two-dimensional study (Mouschovias 1974; Basu et al. 1996, 1997). Other numerical works on the Parker instability include two-dimensional simulations of the undular mode by Matsumoto et al. $(1988,1990)$ and three-dimensional simulations of the mixed mode by Matsumoto \& Shibata (1992), both of which employed a point-mass-dominated gravity. The point- 
mass-dominated gravity can mimic the linearly increasing part of the Galactic gravity at the solar neighborhood when the $\epsilon$ parameter (the ratio of gravitational energy to thermal plus magnetic energy) becomes as large as about 1000. Their simulations under the gravity correctly demonstrated the nonlinear development of the undular and mixed modes, although their numerical resolution was lower than that of Basu et al. and ours. However, application of their results to the Galactic disk was hampered, because their parameter $(\epsilon=6)$ is too small to model the Galactic gravity.

In $\S 2$, the initial setup and the numerical code are briefly described. The results of the simulations and discussions are presented in $\S 3$.

\section{NUMERICAL SETUP AND CODE}

To describe the local behavior of the Parker instability, we introduce Cartesian coordinates $(x, y, z)$ whose directions are radial, azimuthal, and vertical, respectively. Under a uniform external gravity, $-g \hat{z}$, the initial magnetohydrostatic equilibrium state of a system composed of isothermal gas with sound speed $a$ and unidirectional magnetic field $B_{0}=\hat{y} B_{0}(z)$ is given by

$$
\frac{\rho_{0}(z)}{\rho_{0}(0)}=\frac{B_{0}^{2}(z)}{B_{0}^{2}(0)}=\exp \left(\frac{-z}{H}\right)
$$

where the vertical scale height of the gas is defined as $H=$ $(1+\alpha) a^{2} / g$. As Parker (1966) did, we assume $\alpha[\equiv$ $\left.B_{0}^{2} /\left(8 \pi \rho_{0} a^{2}\right)\right]$ to be uniform initially. We study the case with the initial $\alpha=1$ and the adiabatic index $\gamma=1$.

The computational box has $0 \leq x \leq 12 H, 0 \leq y \leq 12 H$, and $0 \leq z \leq 12 H$, where $12 H$ is the azimuthal wavelength of maximum linear growth (Parker 1966). The periodic boundary condition is enforced at $x=0, x=12 H, y=0$, and $y=12 H$ and the reflection boundary condition at $z=0$ and $z=12 \mathrm{H}$. To initiate the instability in the initial equilibrium state, we add random velocity perturbations. The standard deviation of each velocity component is set to be $10^{-4} a$. Since the three-dimensional mixed mode has a maximum linear growth at infinite wavenumber along the radial direction, high-resolution simulations are required in order to resolve resulting small-scale structures. We have performed simulations using $64^{3}, 128^{3}$, and $256^{3}$ cells, although we report mostly the highest resolution one with $256^{3}$ cells. Physical quantities of length, speed, density, and magnetic field are given in units of the density scale height, $H$; the isothermal sound speed, $a$; the initial density at $z=0, \rho_{0}(0)$; and the initial field strength at $z=0, B_{0}(0)$, respectively. For the typical values of $a=6.4 \mathrm{~km} \mathrm{~s}^{-1}$ and $H=160$ pc (Falgarone \& Lequeux 1973), the resulting time unit, $H / a$, becomes $2.5 \times 10^{7}$ yr. The simulations have been made with a three-dimensional isothermal MHD code based on the explicit, finite-difference TVD (total variation diminishing) scheme (Kim et al. 1998).

\section{RESULTS AND DISCUSSIONS}

Figure 1 shows the time evolution of the logarithmic values of the rms velocity components in the simulations with $128^{3}$ and $256^{3}$ cells. The two solid lines are the maximum linear growth rates of the mixed mode, $\Omega_{\max , m}=0.41$, and the undular mode, $\Omega_{\max , u}=0.34$ (Parker 1966, 1967). The evolution patterns in the two panels agree well, indicating that the simu-

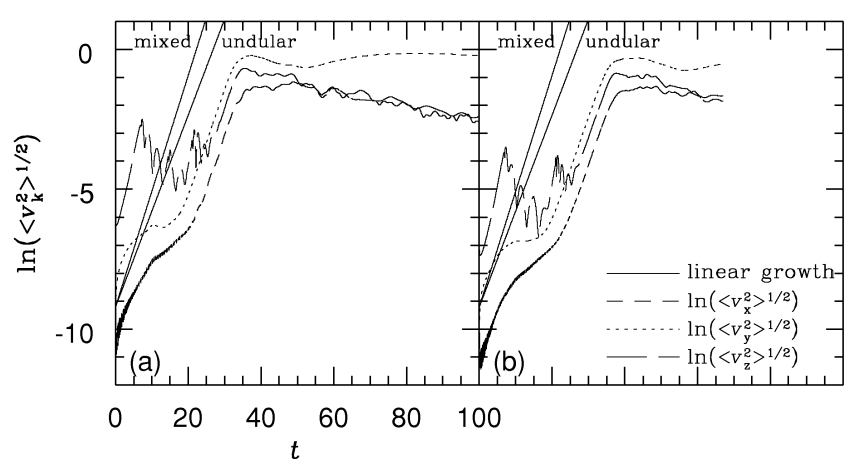

FIG. 1.-Time evolution of the rms values of the radial velocity, $\left\langle v_{x}^{2}\right\rangle^{1 / 2}$, the azimuthal velocity, $\left\langle v_{y}^{2}\right\rangle^{1 / 2}$, and the vertical velocity, $\left\langle v_{z}^{2}\right\rangle^{1 / 2}$. Natural log is used along the ordinate. Left panel, medium-resolution simulation with $128^{3}$ cells; right panel, high-resolution simulation with $256^{3}$ cells. The two solid lines represent the maximum linear growth rates, 0.34 and 0.41 , of the undular mode and the mixed mode, respectively.

lations produce converged results at least for the global quantities such as $\left\langle v^{2}\right\rangle^{1 / 2}$. The evolution is divided into three phases: linear $(0 \leq t \leqq 35)$, nonlinear $(35 \leqq t \leqq 50)$, and relaxed $(t \gtrsim 50)$.

During the initial transient stage of the linear phase $(0 \leq$ $t \lesssim 25$ ), random velocity perturbations adjust themselves by generating a one-dimensional magnetosonic wave, which moves vertically. The peaks in $\left\langle v_{z}^{2}\right\rangle^{1 / 2}$ around $t \simeq 7$ and 21 occur when the wave reaches the upper boundary where the background density is lowest. After the transient stage, the instability grows exponentially $(25 \lesssim t \lesssim 35)$. Kim et al. (1998) showed that in a two-dimensional test simulation the Parker instability grows at the maximum linear growth rate of the undular mode, $\Omega_{\max , u}=0.34$. In Figure 1, however, the Parker instability in three-dimensional simulations grows at a rate that is smaller than the maximum linear growth rate of the mixed mode, although slightly larger than that of the undular mode. This is because the maximum growth of the mixed mode occurs at infinite wavenumber along the radial direction, whereas numerical simulations with finite resolution can not resolve such small structures.

The growth of the instability is slowed down after $t \gtrsim 35$ and saturated in the nonlinear phase because of magnetic tension. The three-dimensional images in Figure 2 depict density structure, velocity vectors, and magnetic field lines at a nonlinear epoch, $t=40$ (Fig. $2 a$ ), as well as at a relaxed epoch, $t=60$ (Fig. $2 b$ ). The two-dimensional images in Figure 3 depict density structure and velocity vectors in a sliced $y-z$ plane, $x-z$ plane, and $x-y$ plane at $t=40$, when the instability is fully developed. Figure $2 a$ shows magnetic arches and valleys created along the magnetic field lines. Their azimuthal wavelength is about 12, that of the undular mode. The structures in the sliced $y-z$ plane of Figure 3 have similarities with those in twodimensional simulations of the undular mode (Basu et al. 1996; Kim et al. 1998). These are the manifestations of the undular mode in the three-dimensional evolution of the Parker instability. On the other hand, the isodensity surface in Figure $2 a$ is corrugated along the $x$-direction. Also, in the sliced $x-z$ and $x-y$ planes, the density and velocity changes rapidly along the direction. These are the manifestations of the interchange mode. The structure has maximum power at the $x$-wavenumbers corresponding to $1 / 16$ to $1 / 32$ of the box size, or eight to 16 cells. This is the size of the smallest structures the code can resolve 

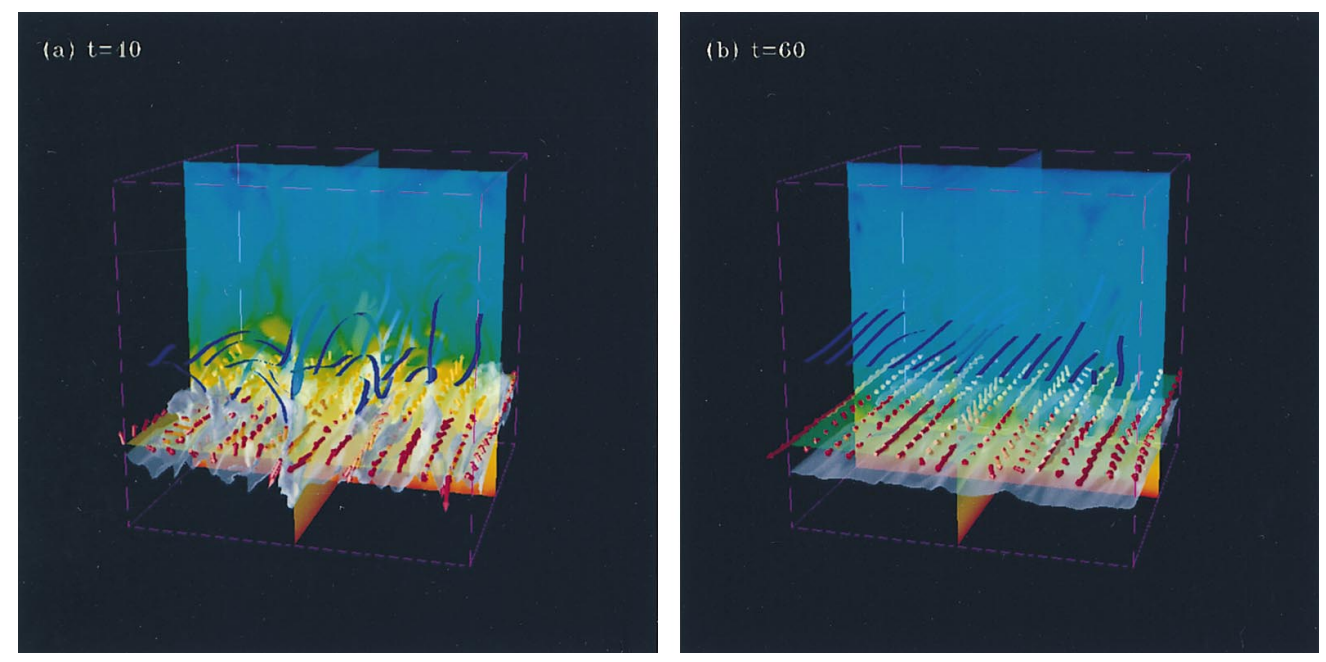

FIG. 2.-Perspective views of density structure, velocity vectors, and the magnetic field lines at two time epochs: $(a) t=40$ and $(b) t=60$, from the highresolution simulation with $256^{3}$ cells. The box is oriented in such a way that the radial $(x)$, the azimuthal $(y)$, and the vertical $(z)$ directions are from left to right, from near to far, and from bottom to top, respectively. Translucent images of density in the three planar slices, $x-y$ plane at $z=3, y-z$ plane at $x=6$, and $x-z$. plane at $y=6$, are overlapped. Colors are mapped from red to blue as density decreases. Gray surface with an isodensity of $\rho_{0}(z=3)$ is included. Velocity field vectors in the $x-y$ plane at $z=3$ are represented by red arrows. Magnetic field lines, whose starting points lie along the line of $z=6$ and $y=0$, are represented by blue ribbons.

cleanly (Ryu, Jones, \& Frank 1995; Kim et al. 1998). Structures smaller than that tend to be dissipated by numerical diffusion. Simulations with higher resolutions would have produced structures with larger $x$-wavenumbers. The comparison between the results of the high-resolution simulation using $256^{3}$ cells and the results of the medium-resolution simulation using $128^{3}$ cells has confirmed the above statement. Hence, we can state that because of the manifestation of the interchange mode, the flow motion in the three-dimensional simulations should be characterized as chaotic. This is different from what was seen in two-dimensional works (Mouschovias 1974; Basu et al. 1996, 1997).

The chaotic flow motion in the nonlinear phase augments magnetic reconnection, especially in the magnetic valley regions (see Jones et al. 1997 for further discussion on reconnection in simulations of this kind). As it accumulates, gas in the valleys is compressed and magnetic field lines are pushed down by the weight of the compressed gas, generating strongly curved field lines. Subsequently, the curved field lines get re- connected, facilitated by the chaotic flow motion. The reconnection allows the gas to drop down off the field lines and at the same time the reconnected field lines to float upward. In this way, gas aggregates along the midplane while the magnetic field is evacuated from it. The chaotic flow motion induces reconnection in other parts of the computational box, but there it contributes less in the redistribution of gas and magnetic field. During the relaxed phase, the dense gas with higher pressure than its surroundings spreads along the magnetic field lines, and the redistributed gas and magnetic field flatten themselves horizontally. A snapshot of the relaxed phase in Figure $2 b$ shows that by $t=60$ the corrugation has been smeared out, the velocity field has been almost aligned with the magnetic field lines, and the magnetic field lines have become more or less straight. However, the isodensity surface, which coincides initially with the $z=3$ plane, has moved downward to $z \simeq 2$. This is an indication that the relaxed system is significantly different from the initial one.

To show quantitatively how much gas is segregated from the

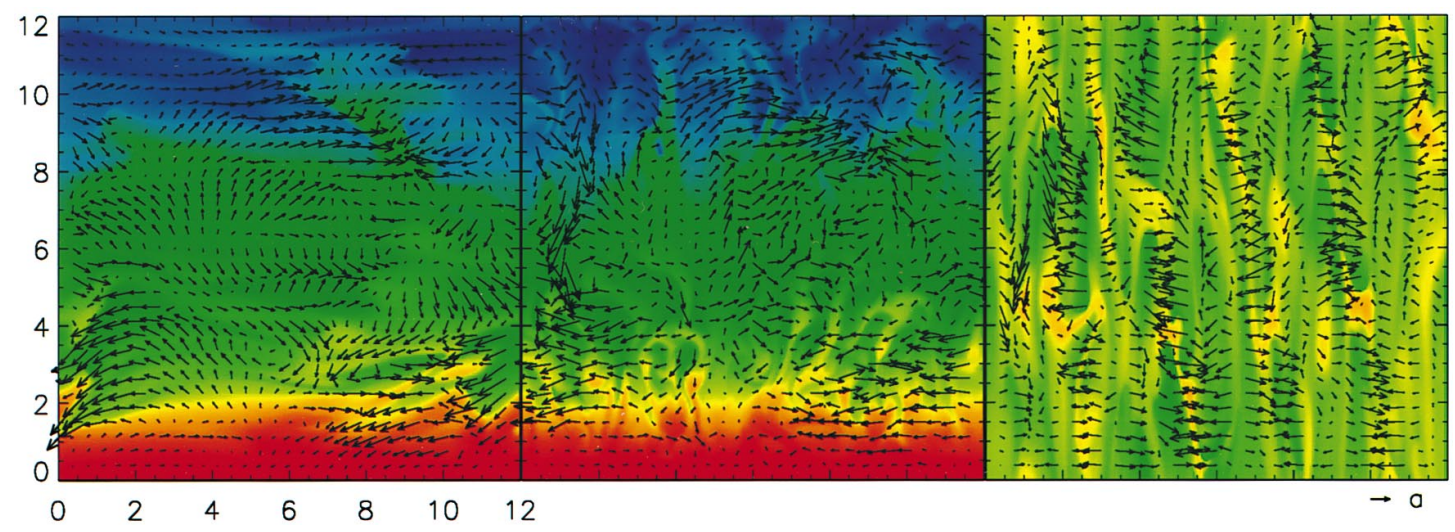

Fig. 3. - Images of density structure and velocity vectors on the three planes, $x=6$ (left), $y=6$ (center), and $z=3$ (right) at $t=40$ from the high-resolution simulation with $256^{3}$ cells. The three planes are same as the sliced planes in Fig. 2. Colors are mapped from red to blue as density decreases. The unit of the velocity vectors is shown at the lower right corner of the image. 


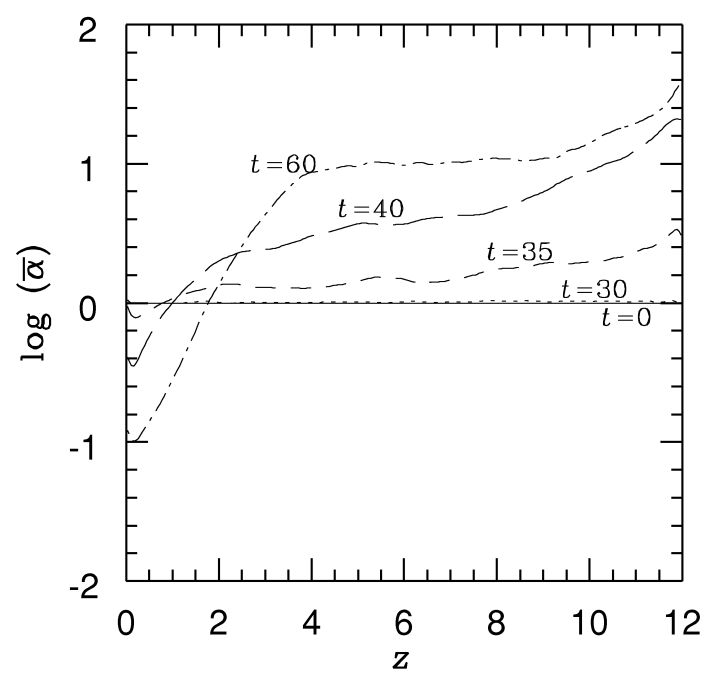

FIG. 4.-The ratio of magnetic to gas pressure $\bar{\alpha}$ averaged over the $x-y$ plane (see eq. [2]) at several time epochs from the high-resolution simulation with $256^{3}$ cells. Common $\log$ is used along the ordinate.

magnetic field, we plot $\bar{\alpha}$, which is defined as

$$
\bar{\alpha}(z ; t)=\int_{0}^{12 H} \int_{0}^{12 H} \frac{B^{2}(x, y, z ; t)}{8 \pi a^{2} \rho(x, y, z ; t)} d x d y \mid \int_{0}^{12 H} \int_{0}^{12 H} d x d y,
$$

with respect to $z$ at several epochs in Figure 4. Initially, $\bar{\alpha}$ is equal to 1 . As time goes by, $\bar{\alpha}$ decreases near the midplane, because the gas aggregates there while the magnetic field is evacuated as stated in the above paragraph. By $t=60, \bar{\alpha}$ decreases by a factor of 10 in the midplane. It increases by more than a factor of 10 around the upper boundary. At $t=60$, in the range $0 \leq z \lesssim 4$ the magnetic field is more or less uniform and the external gravity is now supported almost totally by gas pressure. But for $z \geq 4, \sim 90 \%$ of the external gravity is supported by magnetic pressure and the rest by gas pressure.

So, if the Parker instability is allowed to develop into the relaxed phase without being interrupted by other events, such as explosive energy injections by $\mathrm{OB}$ associations in the Galactic plane, the initial unstable Parker system should evolve into a new stable equilibrium state where the external gravity is mostly supported by the gas pressure alone. In other words, one role that the Parker instability can play in the Galactic plane is to reduce the scale height of interstellar clouds while increasing that of interstellar magnetic field.

Energetics in our simulations has been checked through energy plots (not shown in this paper) that are similar to those in Matsumoto et al. $(1988,1990)$. The total energy of the system (see Mouschovias 1974 for definition) stays constant during the linear phase but decreases significantly during the nonlinear phase. This is because the total energy is no longer a conserved quantity in an isothermal flow, when shock waves are generated in the system (Matsumoto et al. 1990).

How long does it take the Parker instability under the uniform gravity to grow? In estimating the elapsed time, the initial transient stage should not be taken into account, since its duration depends on the initial perturbations and can be reduced if we start the simulations with an initial setup prescribed by the linear analysis. The density at magnetic valleys starts to increase significantly only after the initial transient stage. Hence, the duration of the linear phase except the initial transient stage may be regarded as the growth time of the instability in our simulations. It amounts to $\sim 2.5 \times 10^{8} \mathrm{yr}$. What is the density enhancement resulting from the three-dimensional Parker instability? At the end of the linear phase, $t \simeq 35$, the density enhancement factor reaches the maximum value. Later, it slowly decreases. On the scale of GMCs, $\sim 60 \mathrm{pc}$, which corresponds to eight cells in the high-resolution simulation, the maximum density enhancement along the midplane is $\lesssim 2$ at $t=35$.

The Parker instability has been considered as one of the plausible mechanisms for the formation of GMCs as described in $\S 1$. Based on our three-dimensional simulations of the Parker instability under the uniform gravity, we argue the following: The timescale, $\sim 2.5 \times 10^{8} \mathrm{yr}$, required for the development of the instability is somewhat larger than the lifetime $(\sim 3 \times$ $10^{7} \mathrm{yr}$ ) of GMCs (Blitz \& Shu 1980). The timescale can, however, be reduced, if we replace the uniform gravity by a realistic gravity (Kim \& Hong 1998). Therefore, as far as the timescale is concerned, the Parker instability can be regarded a viable formation mechanism of GMCs. However, the structures formed in the three-dimensional simulations are sheetlike or filamentary, and the maximum density enhancement factor in the scale of GMCs is only 2 . Hence, we conclude that it is difficult to regard the Parker instability alone as the formation mechanism of GMCs.

We are grateful to Doctors B.-C. Koo and Y. Lee for comments on the manuscript. Computations in the present work were carried out by using CRAY T3Es at SERI in Korea and at the University of Minnesota Supercomputing Institute in the US. The work by J. K. was supported in part by the Ministry of Science and Technology through Korea Astronomy Observatory grant 97-5400-000. The work by S. S. H. was supported in part by a grant from the Korea Research Foundation made in the year of 1997. The work by D. R. was supported in part by KOSEF through grant 981-0203-011-2. The work by T. W. J was supported in part by NSF grants AST-9619438 and INT9511654 and by the University of Minnesota Supercomputing Institute.

\section{REFERENCES}

Basu, S., Mouschovias, T. Ch., \& Paleologou, E. V. 1996, Astro. Lett. Comm., 34,333 1997, ApJ, 480, L55

Blitz, L., \& Shu, F. H. 1980, ApJ, 238, 148

Falgarone, E., \& Lequeux, J. 1973, A\&A, 25, 253

Hughes, D. W., \& Cattaneo, F. 1987, Geophys. Astrophys. Fluid Dynamics, 39,65

Jones, T. W., Gaalaas, J. B., Ryu, D., \& Frank, A. 1997, ApJ, 482, 230

Kim, J., \& Hong, S. S. 1998, ApJ, in press
Kim, J., Ryu, D., Jones, T. W., \& Hong, S. S. 1998, ApJ, submitted

Matsumoto, R., Horiuchi, T., Hanawa, T., \& Shibata, K. 1990, ApJ, 356, 259

Matsumoto, R., Horiuchi, T., Shibata, K., \& Hanawa, T. 1988, PASJ, 40, 171

Matsumoto, R., \& Shibata, K. 1992, PASJ, 44, 167

Matsumoto, R., Tajima, T., Shibata, K., \& Kaisig, M. 1993, ApJ, 414, 357

Mouschovias, T. Ch. 1974, ApJ, 192, 37

Mouschovias, T. Ch., Shu, F. H., \& Woodward, P. R. 1974, A\&A, 33, 73

Parker, E. N. 1966, ApJ, 145, 811

- 1967, ApJ, 149, 535

Ryu, D., Jones, T. W., \& Frank, A. 1995, ApJ, 452, 785 